1 Hacettepe Journal of Mathematics and Statistics

$\bigcap$ Volume 43 (6) (2014), 953-961

\title{
On partially $\tau$-quasinormal subgroups of finite groups
}

\author{
Changwen $\mathrm{Li}^{*}$, Xuemei Zhang ${ }^{\dagger}$ and Xiaolan $\mathrm{Yi}^{\ddagger}$
}

\begin{abstract}
Let $H$ be a subgroup of a group $G$. We say that: (1) $H$ is $\tau$-quasinormal in $G$ if $H$ permutes with every Sylow subgroup $Q$ of $G$ such that $(|H|,|Q|)=1$ and $\left(|H|,\left|Q^{G}\right|\right) \neq 1 ;(2) H$ is partially $\tau$-quasinormal in $G$ if $G$ has a normal subgroup $T$ such that $H T$ is $S$-quasinormal in $G$ and $H \cap T \leq H_{\tau G}$, where $H_{\tau G}$ is the subgroup generated by all those subgroups of $H$ which are $\tau$-quasinormal in $G$. In this paper, we find a condition under which every chief factor of $G$ below a normal subgroup $E$ of $G$ is cyclic by using the partial $\tau$-quasinormality of some subgroups.
\end{abstract}

2000 AMS Classification: 20D10, 20D20.

Keywords: $S$-quasinormal, partially $\tau$-quasinormal, cyclic.

Received 05: $06: 2013$ : Accepted $03: 10: 2013$ Doi : 10.15672/HJMS.2104437528

\section{Introduction}

All groups considered in the paper are finite. The notations and terminology in this paper are standard, as in [4] and [6]. $G$ always denotes a finite group, $\pi(G)$ denotes the set of all prime dividing $|G|$ and $F^{*}(G)$ is the generalized Fitting subgroup of $G$, i.e., the product of all normal quasinilpotent subgroups of $G$.

Normal subgroup plays an important role in the study of the structure of groups. Many authors are interested to extend the concept of normal subgroup. For example, a subgroup $H$ of $G$ is said to be $S$-quasinormal [7] in $G$ if $H$ permutes with every Sylow subgroup of $G$. As a generalization of $S$-quasinormality, a subgroup $H$ of $G$ is said to be $\tau$-quasinormal [11] in $G$ if $H$ permutes with every Sylow subgroup $Q$ of $G$ such that $(|H|,|Q|)=1$ and $\left(|H|,\left|Q^{G}\right|\right) \neq 1$. On the other hand, Wang [17] extended normality as

*School of Mathematics and Statistics, Jiangsu Normal University, Xuzhou, 221116, China Email: 1cw2000@126.com

†Department of Basic Sciences, Yancheng Institute of Technology, Yancheng, 224051, China

${ }^{\ddagger}$ School of Science, Zhejiang Sci-Tech University, Hangzhou, 310018, China

The project is supported by the Natural Science Foundation of China (No:11401264) and the Priority Academic Program Development of Jiangsu Higher Education Institutions. 
follows: a subgroup $H$ of $G$ is said to be $c$-normal in $G$ if there exists a normal subgroup $K$ of $G$ such that $H K=G$ and $H \cap K \leq H_{G}$, where $H_{G}$ is the maximal normal subgroup of $G$ contained in $H$. In the literature, many people have studied the influence of the $\tau$-quasinormality and $c$-normality on the structure of finite groups and obtained many interesting results (see $[2,5,8,11,12,17,19]$ ). As a development, we now introduce a new concept:

1.1. Definition. A subgroup $H$ of a group $G$ is said to be partially $\tau$-quasinormal in $G$ if there exists a normal subgroup $T$ of $G$ such that $H T$ is $S$-quasinormal in $G$ and $H \cap T \leq H_{\tau G}$, where $H_{\tau G}$ is the subgroup generated by all those subgroups of $H$ which are $\tau$-quasinormal in $G$.

Clearly, partially $\tau$-quasinormal subgroup covers both the concepts of $\tau$-quasinormal subgroup and $c$-normal subgroup. However, the following examples show that the converse is not true.

1.2. Example. Let $G=S_{4}$ be the symmetric group of degree 4 .

(1) Let $H$ be a Sylow 3-subgroup of $G$ and $N$ the normal abelian 2-subgroup of $G$ of order 4. Then $H N=A_{4} \unlhd G$ and $H \cap N=1$. Hence $H$ is a partially $\tau$-quasinormal subgroup of $G$. But, obviously, $H$ is not $c$-normal in $G$.

(2) Let $H=\langle(14)\rangle$. Obviously, $H A_{4}=G$ and $H \cap A_{4}=1$. Hence $H$ is partially $\tau$ quasinormal in $G$. But, obviously, $H$ is not $\tau$-quasinormal in $G$.

A normal subgroup $E$ of a group $G$ is said to be hypercyclically embedded in $G$ if every chief factor of $G$ below $E$ is cyclic. The product of all normal hypercyclically embedded subgroups of $G$ is denoted by $Z_{\mathscr{U}}(G)$. In [15] and [16], Skiba gave some characterizations of normal hypercyclically embedded subgroups related to $S$-quasinormal subgroups. The main purpose of this paper is to give a new characterization by using partially $\tau$-quasinormal property of maximal subgroups of some Sylow subgroups. We obtain the following result.

Main Theorem. Let $E$ be a normal subgroup of $G$. Suppose that there exists a normal subgroup $X$ of $G$ such that $F^{*}(E) \leq X \leq E$ and $X$ satisfies the following properties: for every non-cyclic Sylow $p$-subgroup $P$ of $X$, every maximal subgroup of $P$ not having a supersoluble supplement in $G$ is partially $\tau$-quasinormal in $G$. Then $E$ is hypercyclically embedded in $G$.

The following theorems are the main stages in the proof of Main Theorem.

1.3. Theorem. Let $P$ be a Sylow $p$-subgroup of a group $G$, where $p$ is a prime divisor of $|G|$ with $(|G|, p-1)=1$. If every maximal subgroup of $P$ not having a $p$-nilpotent supplement in $G$ is partially $\tau$-quasinormal in $G$, then $G$ is soluble.

1.4. Theorem. Let $P$ be a Sylow $p$-subgroup of a group $G$, where $p$ is a prime divisor of $|G|$ with $(|G|, p-1)=1$. Then $G$ is $p$-nilpotent if and only if every maximal subgroup of $P$ not having a $p$-nilpotent supplement in $G$ is partially $\tau$-quasinormal in $G$.

1.5. Theorem. Let $E$ be a normal subgroup in $G$ and let $P$ be a Sylow $p$-subgroup of $E$, where $p$ is a prime divisor of $|E|$ with $(|E|, p-1)=1$. Suppose that every maximal subgroup of $P$ not having a $p$-supersoluble supplement in $G$ is partially $\tau$-quasinormal in $G$. Then each chief factor of $G$ between $E$ and $O_{p^{\prime}}(E)$ is cyclic.

1.6. Theorem. Let $E$ be a normal subgroup of a group $G$. Suppose that for each $p \in \pi(E)$, every maximal subgroup of non-cyclic Sylow $p$-subgroup $P$ of $E$ not having a $p$-supersoluble supplement in $G$ is partially $\tau$-quasinormal in $G$. Then every chief factor of $G$ below $E$ is cyclic. 


\section{Preliminaries}

2.1. Lemma ( [3] and [7]). Suppose that $H$ is a subgroup of $G$ and $H$ is $S$-quasinormal in $G$. Then

(1) If $H \leq K \leq G$, then $H$ is $S$-quasinormal in $K$.

(2) If $N$ is a normal subgroup of $G$, then $H N$ is $S$-quasinormal in $G$ and $H N / N$ is $S$-quasinormal in $G / N$.

(3) If $K \leq G$, then $H \cap K$ is $S$-quasinormal in $K$.

(4) $H$ is subnormal in $G$.

(5) If $K \leq G$ and $K$ is $S$-quasinormal in $G$, then $H \cap K$ is $S$-quasinormal in $G$.

2.2. Lemma ([11, Lemmas 2.2 and 2.3]). Let $G$ be a group and $H \leq K \leq G$.

(1) If $H$ is $\tau$-quasinormal in $G$, then $H$ is $\tau$-quasinormal in $K$.

(2) Suppose that $H$ is normal in $G$ and $\pi(K / H)=\pi(K)$. If $K$ is $\tau$-quasinormal in $G$, then $K / H$ is $\tau$-quasinormal in $G / H$.

(3) Suppose that $H$ is normal in $G$. Then $E H / H$ is $\tau$-quasinormal in $G / H$ for every $\tau$-quasinormal subgroup $E$ in $G$ satisfying $(|H|,|E|)=1$.

(4) If $H$ is $\tau$-quasinormal in $G$ and $H \leq O_{p}(G)$ for some prime $p$, then $H$ is $S$ quasinormal in $G$.

(5) $H_{\tau G} \leq H_{\tau K}$.

(6) Suppose that $K$ is a $p$-group and $H$ is normal in $G$. Then $K_{\tau G} / H \leq(K / H)_{\tau(G / H)}$.

(7) Suppose that $H$ is normal in $G$. Then $E_{\tau G} H / H \leq(E H / H)_{\tau(G / H)}$ for every p-subgroup $E$ of $G$ satisfying $(|H|,|E|)=1$.

2.3. Lemma. Let $G$ be a group and $H \leq G$. Then

(1) If $H$ is partially $\tau$-quasinormal in $G$ and $H \leq K \leq G$, then $H$ is partially $\tau$ quasinormal in $K$.

(2) Suppose that $N \unlhd G$ and $N \leq H$. If $H$ is a $p$-group and $H$ is partially $\tau$ quasinormal in $G$, then $H / N$ is partially $\tau$-quasinormal in $G / N$.

(3) Suppose that $H$ is a $p$-subgroup of $G$ and $N$ is a normal $p^{\prime}$-subgroup of $G$. If $H$ is partially $\tau$-quasinormal in $G$, then $H N / N$ is partially $\tau$-quasinormal in $G / N$.

(4) If $H$ is partially $\tau$-quasinormal in $G$ and $H \leq K \unlhd G$, then there exists $T \unlhd G$ such that $H T$ is $S$-quasinormal in $G, H \cap T \leq H_{\tau G}$ and $\bar{H} T \leq K$.

Proof. (1) Let $N$ be a normal subgroup of $G$ such that $H N$ is $S$-quasinormal in $G$ and $H \cap N \leq H_{\tau G}$. Then $K \cap N \unlhd K, H(K \cap N)=H N \cap K$ is $S$-quasinormal in $K$ by Lemma 2.1(3) and $H \cap(K \cap N)=H \cap N \leq H_{\tau G} \leq H_{\tau K}$ by Lemma 2.2(5). Hence $H$ is partially $\tau$-quasinormal in $K$.

(2) Suppose that $H$ is partially $\tau$-quasinormal in $G$. Then there exists $K \unlhd G$ such that $H K$ is $S$-quasinormal in $G$ and $H \cap K \leq H_{\tau G}$. This implies that $K N / N \unlhd G / N$ and $(H / N)(K N / N)=H K / N$ is $S$-quasinormal in $G / N$ by Lemma 2.1(2). In view of Lemma 2.2(6), $H / N \cap K N / N=(H \cap K) N / N \leq H_{\tau G} N / N=H_{\tau G} / N \leq(H / N)_{\tau(G / N)}$. Thus $H / N$ is partially $\tau$-quasinormal in $G / N$.

(3) Suppose that $H$ is partially $\tau$-quasinormal in $G$. Then there exists $K \unlhd G$ such that $H K$ is $S$-quasinormal in $G$ and $H \cap K \leq H_{\tau G}$. Clearly, $K N / N \unlhd G$ and $(H N / N)(K N / N)=H K N / N$ is $S$-quasinormal in $G / N$ by Lemma 2.1(2). On the other hand, since $(|H N: H|,|H N: N|)=1, H N / N \cap K N / N=(H N \cap K) N / N=$ $(H \cap K)(N \cap K) N / N=(H \cap K) N / N \leq H_{\tau G} N / N$. In view of Lemma 2.2(7), we have $H_{\tau G} N / N \leq(H N / N)_{\tau(G / N)}$. Hence $H N / N$ is partially $\tau$-quasinormal in $G / N$.

(4) Suppose that $H$ is partially $\tau$-quasinormal in $G$. Then there exists $N \unlhd G$ such that $H N$ is $S$-quasinormal in $G$ and $H \cap N \leq H_{\tau G}$. Let $T=N \cap K$. Then $T \unlhd G$, $H T=H(N \cap K)=H N \cap K$ is $S$-quasinormal in $G$ by Lemma 2.1(5), $H T \leq K$ and $H \cap T=H \cap N \cap K=H \cap N \leq H_{\tau G}$. 
2.4. Lemma. Let $G$ be a group and $p$ a prime dividing $|G|$ with $(|G|, p-1)=1$.

(1) If $N$ is normal in $G$ of order $p$, then $N$ lies in $Z(G)$.

(2) If $G$ has cyclic Sylow $p$-subgroups, then $G$ is $p$-nilpotent.

(3) If $M \leq G$ and $|G: M|=p$, then $M \unlhd G$.

(4) If $G$ is $p$-supersoluble, then $G$ is $p$-nilpotent.

Proof. (1), (2) and (3) can be found in [18, Theorem 2.8]. Now we only prove (4). Let $A / B$ be an arbitrary chief factor of $G$. If $G$ is $p$-supersolvable, then $A / B$ is either a cyclic group with order $p$ or a $p^{\prime}$-group. If $|A / B|=p$, then $|\operatorname{Aut}(A / B)|=p-1$. Since $G / C_{G}(A / B)$ is isomorphic to a subgroup of $\operatorname{Aut}\left((A / B)\right.$, the order of $G / C_{G}(A / B)$ must divide $(|G|, p-1)=1$, which shows that $G=C_{G}(A / B)$. Therefore, we have $G$ is $p$-nilpotent.

2.5. Lemma ([10, Lemma 2.12]). Let $P$ be a Sylow $p$-subgroup of a group $G$, where $p$ is a prime divisor of $|G|$ with $(|G|, p-1)=1$. If every maximal subgroup of $P$ has a $p$-nilpotent supplement in $G$, then $G$ is $p$-nilpotent.

2.6. Lemma ([13, Theorem A]). If $P$ is an $S$-quasinormal $p$-subgroup of a group $G$ for some prime $p$, then $N_{G}(P) \geq O^{p}(G)$.

2.7. Lemma ([6, VI, 4.10]). Assume that $A$ and $B$ are two subgroups of a group $G$ and $G \neq A B$. If $A B^{g}=B^{g} A$ holds for any $g \in G$, then either $A$ or $B$ is contained in a nontrivial normal subgroup of $G$.

2.8. Lemma ([20, Chap.1, Theorem 7.19]). Let $H$ be a normal subgroup of $G$. Then $H \leq Z_{\mathscr{U}}(G)$ if and only if $H / \Phi(H) \leq Z_{\mathscr{U}}(G / \Phi(H))$.

2.9. Lemma ([14, Lemma 2.11]). Let $N$ be an elementary abelian normal subgroup of a group $G$. Assume that $N$ has a subgroup $D$ such that $1<|D|<|N|$ and every subgroup $H$ of $N$ satisfying $|H|=|D|$ is $S$-quasinormal in $G$. Then some maximal subgroup of $N$ is normal in $G$.

2.10. Lemma. Let $N$ be a non-identity normal $p$-subgroup of a group $G$. If $N$ is elementary and every maximal subgroup of $N$ is partially $\tau$-quasinormal in $G$, then some maximal subgroup of $N$ is normal in $G$.

Proof. If $|N|=p$, then it is clear. Let $L$ be a non-identity minimal normal $p$-subgroup of $G$ contained in $N$. First we assume that $N \neq L$. By Lemma 2.3(2), the hypothesis still holds on $G / L$. Then by induction some maximal subgroup $M / L$ of $N / L$ is normal in $G / L$. Clearly, $M$ is a maximal subgroup of $N$ and $M$ is normal in $G$. Consequently the lemma follows. Now suppose that $L=N$. Let $M$ be any maximal subgroup of $N$. Then by the hypothesis, there exists $T \unlhd G$ such that $M T$ is $S$-quasinormal in $G$ and $M \cap T \leq M_{\tau G}$. Suppose that $M \neq M_{\tau G}$. Then $M T \neq M$ and $T \neq 1$. If $N \leq M T$, then $N=N \cap M T=M(N \cap T)$. Hence $N \leq T$, which implies that $M=M \cap T=M_{\tau G}$, a contradiction. If $N \nsubseteq M T$, then $M=M(T \cap N)=M T \cap N$ is $S$-quasinormal in $G$ by Lemma 2.1(5), a contradiction again. Hence $M=M_{\tau G}$. In view of Lemma 2.2(4), $M$ is $S$-quasinormal in $G$. By Lemma 2.9, some maximal subgroup of $N$ is normal in $G$. Thus the lemma holds.

2.11. Lemma ([15, Theorem B]). Let $\mathscr{F}$ be any formation and $G$ a group. If $H \triangleleft G$ and $F^{*}(H) \leq Z_{\mathscr{F}}(G)$, then $H \leq Z_{\mathscr{F}}(G)$. 


\section{Proofs of Theorems}

Proof of Theorem 1.3. Assume that this theorem is false and let $G$ be a counterexample with minimal order. We proceed the proof via the following steps.

(1) $O_{p}(G)=1$.

Assume that $L=O_{p}(G) \neq 1$. Clearly, $P / L$ is a Sylow $p$-subgroup of $G / L$. Let $M / L$ be a maximal subgroup of $P / L$. Then $M$ is a maximal subgroup of $P$. If $M$ has a $p$-nilpotent supplement $D$ in $G$, then $M / L$ has a $p$-nilpotent supplement $D L / L$ in $G / L$. If $M$ is partially $\tau$-quasinormal in $G$, then $M / L$ is partially $\tau$-quasinormal in $G / L$ by Lemma 2.3(2). Hence $G / L$ satisfies the hypothesis of the theorem. The minimal choice of $G$ implies that $G / L$ is soluble. Consequently, $G$ is soluble. This contradiction shows that step (1) holds.

(2) $O_{p^{\prime}}(G)=1$.

Assume that $R=O_{p^{\prime}}(G) \neq 1$. Then, obviously, $P R / R$ is a Sylow $p$-subgroup of $G / R$. Suppose that $M / R$ is a maximal subgroup of $P R / R$. Then there exists a maximal subgroup $P_{1}$ of $P$ such that $M=P_{1} R$. If $P_{1}$ has a $p$-nilpotent supplement $D$ in $G$, then $M / R$ has a $p$-nilpotent supplement $D R / R$ in $G / R$. If $P_{1}$ is partially $\tau$-quasinormal in $G$, then $M / R$ is partially $\tau$-quasinormal in $G / R$ by Lemma $2.3(3)$. The minimal choice of $G$ implies that $G / R$ is soluble. By the well known Feit-Thompson's theorem, we know that $R$ is soluble. It follows that $G$ is soluble, a contradiction.

(3) $P$ is not cyclic.

If $P$ is cyclic, then $G$ is $p$-nilpotent by Lemma 2.4 , and so $G$ is soluble, a contradiction.

(4) If $N$ is a minimal normal subgroup of $G$, then $N$ is not soluble. Moreover, $G=P N$.

If $N$ is $p$-soluble, then $O_{p}(N) \neq 1$ or $O_{p^{\prime}}(N) \neq 1$. Since $O_{p}(N)$ char $N \unlhd G$, $O_{p}(N) \leq O_{p}(G)$. Analogously $O_{p^{\prime}}(N) \leq O_{p^{\prime}}(G)$. Hence $O_{p}(G) \neq 1$ or $O_{p^{\prime}}(G) \neq 1$, which contradicts step (1) or step (2). Therefore $N$ is not soluble. Assume that $P N<G$. By Lemma 2.3(1), every maximal subgroup of $P$ not having a $p$-nilpotent supplement in $P N$ is partially $\tau$-quasinormal in $P N$. Thus $P N$ satisfies the hypothesis. By the minimal choice of $G, P N$ is soluble and so $N$ is soluble. This contradiction shows that $G=P N$.

(5) $G$ has a unique minimal normal subgroup $N$.

By step (4), we see that $G=P N$ for every normal subgroup $N$ of $G$. It follows that $G / N$ is soluble. Since the class of all soluble groups is closed under subdirect product, $G$ has a unique minimal normal subgroup, say $N$.

(6) The final contradiction.

If every maximal subgroup of $P$ has a $p$-nilpotent supplement in $G$, then, in view of Lemma 2.5, $G$ is $p$-nilpotent and so $G$ is soluble. This contradiction shows that we may choose a maximal subgroup $P_{1}$ of $P$ such that $P_{1}$ is partially $\tau$-quasinormal in $G$. Then there exists a normal subgroup $T$ of $G$ such that $P_{1} T$ is $S$-quasinormal in $G$ and $P_{1} \cap T \leq\left(P_{1}\right)_{\tau G}$. If $T=1$, then $P_{1}$ is $S$-quasinormal in $G$. In view of Lemma 2.6, $P_{1} \unlhd \bar{P} O^{p}(G)=G$. By step (5), $P_{1}=1$ or $N \leq P_{1}$. Since $N$ is not soluble by step (4), we have that $P_{1}=1$. Consequently, $P$ is cyclic, which contradicts step (3). Hence $T \neq 1$ and $N \leq T$. It follows that $P_{1} \cap N=\left(P_{1}\right)_{\tau G} \cap N$. For any Sylow $q$-subgroup $N_{q}$ of $N$ with $q \neq p, N_{q}$ is also a Sylow $q$-subgroup of $G$ by step (4). From step (2) it is easy to see that $\left(P_{1}\right)_{\tau G} N_{q}=N_{q}\left(P_{1}\right)_{\tau G}$. Then $\left(P_{1}\right)_{\tau G} N_{q} \cap N=N_{q}\left(\left(P_{1}\right)_{\tau G} \cap N\right)=N_{q}\left(P_{1} \cap N\right)$, i.e., $P_{1} \cap N$ is $\tau$-quasinormal in $N$. Since $N$ is a direct product of some isomorphic non-abelian simple groups, we may assume that $N \cong N_{1} \times \cdots \times N_{k}$. By Lemma 2.2(1), $P_{1} \cap N$ is $\tau$-quasinormal in $\left(P_{1} \cap N\right) N_{1}$. Thus $\left(P_{1} \cap N\right)\left(N_{1 q}\right)^{n_{1}} \cap N_{1}=\left(N_{1 q}\right)^{n_{1}}\left(P_{1} \cap N \cap N_{1}\right)=\left(N_{1 q}\right)^{n_{1}}\left(P_{1} \cap N_{1}\right)$ for any $n_{1} \in N_{1}$, where $N_{1 q}$ is a Sylow $q$-subgroup of $N_{1}$ with $q \neq p$. Since $\left(N_{1 q}\right)^{n_{1}}\left(P_{1} \cap N_{1}\right) \neq N_{1}$, we have $N_{1}$ is not simple by Lemma 2.7, a contradiction. 
Proof of Theorem 1.4. If $G$ is $p$-nilpotent, then $G$ has a normal Hall $p^{\prime}$-subgroup $G_{p^{\prime}}$. Let $P_{1}$ be any maximal subgroup of $P$. Then $\left|G: P_{1} G_{p^{\prime}}\right|=p$. In view of Lemma 2.4(3), $P_{1} G_{p^{\prime}} \unlhd G$. Obviously, $P_{1} \cap G_{p^{\prime}}=1$. Hence $P_{1}$ is partially $\tau$-quasinormal in $G$.

Now we prove the sufficient part. Assume that the assertion is false and let $G$ be a counterexample with minimal order.

(1) $G$ is soluble.

It follows directly from Theorem 1.3.

(2) $G$ has a unique minimal normal subgroup $N$ such that $G / N$ is $p$-nilpotent. Moreover, $\Phi(G)=1$.

Let $N$ be a minimal normal subgroup of $G$. Since $G$ is solvable by step (1), $N$ is an elementary abelian subgroup. It is easy to see that $G / N$ satisfies the hypothesis of our theorem by Lemma 2.3. By the minimal choice of $G, G / N$ is $p$-nilpotent. Since the class of all $p$-nilpotent groups is a saturated formation, $N$ is a unique minimal normal subgroup of $G$ and $\Phi(G)=1$.

(3) $P$ is not cyclic.

If $P$ is cyclic, $G$ is $p$-nilpotent by Lemma $2.4(2)$, a contradiction.

(4) $O_{p^{\prime}}(G)=1$.

(5) Every maximal subgroup of $P$ has a $p$-nilpotent supplement in $G$.

It is clear that $N \leq O_{p}(G)$. By $\Phi(G)=1$, we may choose a maximal subgroup $M$ of $G$ such that $G=N M$ and $G / N \cong M$. Let $P_{1}$ be an arbitrary maximal subgroup of $P$. We will show $P_{1}$ has a $p$-nilpotent supplement in $G$. Since $N$ has the $p$-nilpotent supplement $M$ in $G$, we only need to prove $N \leq P_{1}$ when $P_{1}$ is partially $\tau$-quasinormal in $G$. Let $T$ be a normal subgroup of $G$ such that $P_{1} T$ is $S$-quasinormal in $G$ and $P_{1} \cap T \leq\left(P_{1}\right)_{\tau G}$. First, we assume that $T=1$, i.e., $P_{1}$ is $S$-quasinormal in $G$. In view of Lemma 2.6, $P_{1} \unlhd P O^{p}(G)=G$. By virtue of Lemma 2.4(2) and step (3), $P_{1} \neq 1$. Hence $N \leq P_{1}$ by step (2). Now, assume that $T \neq 1$. Then $N \leq T$. It follows that $P_{1} \cap N=\left(P_{1}\right)_{\tau G} \cap N$. For any Sylow $q$-subgroup $G_{q}$ of $G(p \neq q),\left(P_{1}\right)_{\tau G} G_{q}=G_{q}\left(P_{1}\right)_{\tau G}$ in view of step (4). Then $\left(P_{1}\right)_{\tau G} \cap N=\left(P_{1}\right)_{\tau G} G_{q} \cap N \unlhd\left(P_{1}\right)_{\tau G} G_{q}$. Obviously, $P_{1} \cap N \unlhd P$. Therefore $P_{1} \cap N$ is normal in $G$. By the minimality of $N$, we have $P_{1} \cap N=N$ or $P_{1} \cap N=1$. If the later holds, then the order of $N$ is $p$ since $P_{1} \cap N$ is a maximal subgroup of $N$. Consequently, $G$ is $p$-nilpotent by step (2) and Lemma 2.4(1). This contradiction shows that $P_{1} \cap N=N$ and so $N \leq P_{1}$.

(6) The final contradiction.

Since every maximal subgroup of $P$ has a $p$-nilpotent supplement in $G$ by step (5), we have $G$ is $p$-nilpotent by Lemma 2.5 , a contradiction.

Proof of Theorem 1.5. Assume that this theorem is false and and consider a counterexample $(G, E)$ for which $|G||E|$ is minimal.

(1) $E$ is $p$-nilpotent.

Let $P_{1}$ be a maximal subgroup of $P$. If $P_{1}$ has a $p$-supersolvable supplement $T$ in $G$, then $P_{1}$ has a $p$-supersolvable supplement $T \cap E$ in $E$. Since $(|E|, p-1)=1, T \cap E$ is also $p$-nilpotent by Lemma $2.4(4)$. If $P_{1}$ is partially $\tau$-quasinormal in $G$, then $P_{1}$ is also partially $\tau$-quasinormal in $E$ by Lemma $2.3(1)$. Hence every maximal subgroup of $P$ not having a $p$-nilpotent supplement in $E$ is partially $\tau$-quasinormal in $E$. In view of Theorem 1.4, $E$ is $p$-nilpotent.

(2) $P=E$.

By step (1), $O_{p^{\prime}}(E)$ is the normal Hall $p^{\prime}$-subgroup of $E$. Suppose that $O_{p^{\prime}}(E) \neq 1$. It is easy to see that the hypothesis of the theorem holds for $\left(G / O_{p^{\prime}}(E), E / O_{p^{\prime}}(E)\right)$. By induction, every chief factor of $G / O_{p^{\prime}}(E)$ between $E / O_{p^{\prime}}(E)$ and 1 is cyclic. Consequently, each chief factor of $G$ between $E$ and $O_{p^{\prime}}(E)$ is cyclic. This condition shows that $O_{p^{\prime}}(E)=1$ and so $P=E$. 
(3) $\Phi(P)=1$.

Suppose that $\Phi(P) \neq 1$. By Lemma 2.3(2), it is easy to see that the hypothesis of the theorem holds for $(G / \Phi(P), P / \Phi(P))$. By the choice of $(G, E)$, every chief factor of $G / \Phi(P)$ below $P / \Phi(P)$ is cyclic. In view of Lemma 2.8, every chief factor of $G$ below $P$ is cyclic, a contradiction.

(4) Every maximal subgroup of $P$ is partially $\tau$-quasinormal in $G$.

Suppose that there is some maximal subgroup $V$ of $P$ such that $V$ has a $p$-supersolvable supplement $B$ in $G$, then $G=P B$ and $P \cap B \neq 1$. Since $P \cap B \unlhd B$, we may assume that $B$ has a minimal normal subgroup $N$ contained in $P \cap B$. It is clear that $|N|=p$. Since $P$ is elementary abelian and $G=P B$, we have that $N$ is also normal in $G$. It is easy to see that the hypothesis is still true for $(G / N, P / N)$. Hence every chief factor of $G / N$ below $P / N$ is cyclic by virtue of the choice of $(G, E)$. It follows that every chief factor of $G$ below $P$ is cyclic. This contradiction shows that all maximal subgroups of $P$ are partially $\tau$-quasinormal in $G$.

(5) $P$ is not a minimal normal subgroup of $G$.

Suppose that $P$ is a minimal normal subgroup of $G$, then some maximal subgroup of $P$ is normal in $G$ by Lemma 2.10, which contradicts the minimality of $P$.

(6) If $N$ is a minimal normal subgroup of $G$ contained in $P$, then $P / N \leq Z_{\mathscr{U}}(G / N)$, $N$ is the only minimal normal subgroup of $G$ contained in $P$ and $|N|>p$.

Indeed, by Lemma $2.3(2)$, the hypothesis holds on $(G / N, P / N)$ for any minimal normal subgroup $N$ of $G$ contained in $P$. Hence every chief factor of $G / N$ below $P / N$ is cyclic by the choice of $(G, E)=(G, P)$. If $|N|=p$, every chief factor of $G$ below $P$ is cyclic, a contradiction. If $G$ has two minimal normal subgroups $R$ and $N$ contained in $P$, then $N R / R \leq P / R$ and from the $G$-isomorphism $N R / R \cong N$ we have $|N|=p$, a contradiction. Hence, (6) holds.

(7) The final contradiction.

Let $N$ be a minimal normal subgroup of $G$ contained in $P$ and $N_{1}$ any maximal subgroup of $N$. We show that $N_{1}$ is $S$-quasinormal in $G$. Since $P$ is an elementary abelian $p$-group, we may assume that $D$ is a complement of $N$ in $P$. Let $V=N_{1} D$. Obviously, $V$ is a maximal subgroup of $P$. By step (4), $V$ is partially $\tau$-quasinormal in $G$. By Lemma 2.3(4), there exist a normal subgroup $T$ of $G$ such that $V T$ is $S$ quasinormal in $G, V \cap T \leq V_{\tau G}$ and $V T \leq P$. In view of Lemma 2.2(4), $V_{\tau G}$ is an $S$-quasinormal subgroup of $G$. If $T=P$, then $V=V_{\tau G}$ is $S$-quasinormal in $G$ and hence $V \cap N=N_{1} D \cap N=N_{1}(D \cap N)=N_{1}$ is $S$-quasinormal in $G$ by Lemma 2.1(5). If $T=1$, then $V=V T$ is $S$-quasinormal in $G$. Consequently, we have also $N_{1}$ is $S$ quasinormal in $G$. Now we assume that $1<T<P$. Hence $N \leq T$ by step (6). Then, $N_{1}=V \cap N=V_{\tau G} \cap N$ is $S$-quasinormal in $G$ by virtue of Lemma 2.1(5). Hence some maximal subgroup of $N$ is normal in $G$ by Lemma 2.9. Consequently, $|N|=p$. This contradicts step (6).

Proof of Theorem 1.6. Let $q$ be the smallest prime dividing $|E|$. In view of step (1) of the proof of Theorem 1.5, $E$ is $q$-nilpotent. Let $E_{q^{\prime}}$ be the normal Hall $q^{\prime}$-subgroup of $E$. If $E_{q^{\prime}}=1$, then every chief factor of $G$ below $E$ is cyclic by Theorem 1.5. Hence we may assume that $E_{q^{\prime}} \neq 1$. Since $E_{q^{\prime}}$ char $E \unlhd G$, we see that $E_{q^{\prime}} \unlhd G$. By Lemma 2.3(3), the hypothesis of the theorem holds for $\left(G / E_{q^{\prime}}, E / E_{q^{\prime}}\right)$. By induction, every chief factor of $G / E_{q^{\prime}}$ below $E / E_{q^{\prime}}$ is cyclic. On the other hand, $\left(G, E_{q^{\prime}}\right)$ also satisfies the hypothesis of the theorem in view of Lemma 2.3(1). By induction again, we have also every chief factor of $G$ below $E_{q^{\prime}}$ is cyclic. Hence it follows that every chief factor of $G$ below $E$ is cyclic. 
Proof of Main Theorem. Applying Theorem 1.6, $X$ is hypercyclically embedded in $G$. Since $F^{*}(E) \leq X$, we have that $F^{*}(E)$ is also hypercyclically embedded in $G$. By virtue of Lemma $2.11, E$ is also hypercyclically embedded in $G$.

\section{Some Applications}

4.1. Theorem. Let $\mathscr{F}$ be a saturated formation containing $\mathscr{U}$ and $E$ a normal subgroup of a group $G$ such that $G / E \in \mathscr{F}$. Suppose that for every non-cyclic Sylow subgroup $P$ of $E$, every maximal subgroup of $P$ not having a supersoluble supplement in $G$ is partially $\tau$-quasinormal in $G$. Then $G \in \mathscr{F}$.

Proof. Applying our Main Theorem, every chief factor of $G$ below $E$ is cyclic. Since $\mathscr{F}$ contains $\mathscr{U}$, we know $E$ is contained in the $\mathscr{F}$-hypercentre of $G$. From $G / E \in \mathscr{F}$, it follows that $G \in \mathscr{F}$.

4.2. Theorem. Let $\mathscr{F}$ be a saturated formation containing $\mathscr{U}$ and $E$ a normal subgroup of a group $G$ such that $G / E \in \mathscr{F}$. Suppose that for every non-cyclic Sylow subgroup $P$ of $F^{*}(E)$, every maximal subgroup of $P$ not having a supersoluble supplement in $G$ is partially $\tau$-quasinormal in $G$. Then $G \in \mathscr{F}$.

Proof. The proof is similar to that of Theorem 4.1.

4.3. Corollary $([9$, Theorem 3.4$])$. Let $\mathscr{F}$ be a saturated formation containing $\mathscr{U}$ and $E$ a normal subgroup of a group $G$ such that $G / E \in \mathscr{F}$. If every maximal subgroup of any Sylow subgroup of $F^{*}(E)$ is $S$-quasinormal in $G$, then $G \in \mathscr{F}$.

4.4. Corollary ([19, Theorem 3.4]). Let $\mathscr{F}$ be a saturated formation containing $\mathscr{U}$ and $E$ a normal subgroup of a group $G$ such that $G / E \in \mathscr{F}$. If every maximal subgroup of any Sylow subgroup of $F^{*}(E)$ is $c$-normal in $G$, then $G \in \mathscr{F}$.

4.5. Corollary ([1, Theorem 1.4]). Let $\mathscr{F}$ be a saturated formation containing $\mathscr{U}$ and $E$ a soluble normal subgroup of a group $G$ such that $G / E \in \mathscr{F}$. If every maximal subgroup of any Sylow subgroup of $F(E)$ is $S$-quasinormal in $G$, then $G \in \mathscr{F}$.

4.6. Corollary ([8, Theorem 2]). Let $G$ be a group and $E$ a soluble normal subgroup of $G$ such that $G / E$ is supersolvable. If all maximal subgroups of the Sylow subgroups of $F(E)$ are $c$-normal in $G$, then $G$ is supersolvable.

\section{References}

[1] M. Asaad, On maximal subgroups of Sylow subgroups of finite groups, Comm. Algebra, 26(1998), 3647-3652.

[2] A. Ballester-Bolinches and Y. Wang, Finite groups with some $c$-normal minimal subgroups, J. Pure Appl. Algebra, 153 (2000), 121-127.

[3] W. E. Deskins, On quasinormal subgroups of finite groups, Math. Z. 82 (1963), 125-132.

[4] W. Guo, The Theory of Class of Groups, Science Press-Kluwer Academic Publishers, BeijingNew York-Dordrecht-Boston-London, 2000.

[5] X. Guo and K. P. Shum, On $c$-normal maximal and minimal subgroups of Sylow $p$-subgroups of finite groups, Arch. Math. 80 (2003), 561-569.

[6] B. Huppert, Endliche Gruppen I, Springer-Verlag, Berlin-Heidelberg-New York, 1967.

[7] O. H. Kegel, Sylow Gruppen und subnormalteiler endlicher Gruppen, Math. Z. 78 (1962), 205-221.

[8] D. Li and X. Guo, The influence of c-normality of subgroups on the structure of finite groups II, Comm. Algebra, 26 (1998), 1913-1922.

[9] Y. Li, H. Wei and Y. Wang, The influence of $\pi$-quasinormality of some subgroups of a finite griup, Arch. Math. 81 (2003), 245-252. 
[10] C. Li, Finite groups with some primary subgroups $S S$-quasinormally embedded, Indian J. Pure Appl. Math. 42 (2011) 291-306.

[11] V. O. Lukyanenko and A. N. Skiba, On weakly $\tau$-quasinormal subgroups of finite groups, Acta Math. Hungar, 125 (2009), 237-248.

[12] V. O. Lukyanenko and A. N. Skiba, Finite groups in which $\tau$-quasinormality is a transitive relation, Rend Sem Mat Univ Padova, 124 (2010), 231-246.

[13] P. Schmidt, Subgroups permutable with all Sylow subgroups, J. Algebra, 207 (1998), 285293.

[14] A. N. Skiba, On weakly $S$-permutable subgroups of finite groups, J. Algebra, 315 (2007), 192-209.

[15] A. N. Skiba, On two questions of L. A. Shemetkov concerning hypercyclically embedded subgroups of finite groups, J. Group Theory, 13 (2010), 841-850.

[16] A. N. Skiba, Cyclicity conditions for $G$-chief factors of normal subgroups of a group $G$, Siberian Math. J. 52 (2011), 127-130.

[17] Y. Wang, $C$-normality and groups and its properties, J. Algebra, 180 (1996), 954-965.

[18] H. Wei and Y. Wang, On $c^{*}$-normality and its properties, J. Group Theory, 10 (2007), 211-223.

[19] H. Wei, Y. Wang and Y. Li, On $c$-normal maximal and minimal subgroups of Sylow subgroups of finite groups II, Comm. Algebra, 31 (2003), 4807-4816.

[20] M. Weinstein, Between Nilpotent and Solvable, Polygonal Publishing House, Passaic, 1982. 
